\title{
Nonisothermal eutectic crystallization
}

\author{
K. R. Elder ${ }^{1,2}$ J. D. Gunton, ${ }^{3}$ and Martin Grant ${ }^{4}$ \\ ${ }^{1}$ Department of Physics, Oakland University, Rochester, Michigan, 48309-4401 \\ ${ }^{2}$ Department of Physics and Materials Research Laboratory, University of Illinois at Urbana-Champaign, 1110 West Green Street, \\ Urbana, Illinois 61801 \\ ${ }^{3}$ Department of Physics, Lehigh University, 16 Memorial Drive East, Bethlehem, Pennsylvania, 18015-3182 \\ ${ }^{4}$ Department of Physics, McGill University, Montreal, Quèbec, Canada
}

(Received 10 January 1996)

\begin{abstract}
A model for the dynamics of nonisothermal eutectic growth is examined in the limit of a spatially uniform but time dependent temperature field. The simpler case of nonisothermal growth is first considered, and approximate solutions are obtained for the crystallization rate for the growth of a single crystallite and for homogeneous nucleation. For eutectic transformation the latent heat generated during invasion of a eutectic crystal into an amorphous background is shown to increase the lamella wavelength. For homogeneous eutectic growth, the latent heat is shown to alter significantly the growth mechanisms observed under isothermal conditions. [S1063-651X(96)10408-6]
\end{abstract}

PACS number(s): 64.70.Dv, 05.70.Ln, 82.20.Mj

\section{INTRODUCTION}

Eutectics are materials in which a liquid can coexist with two crystal phases of different concentrations at a specific temperature $\left(T_{E}\right)$ and concentration $\left(C_{E}\right)$ that define the eutectic point. The solidification of the liquid phase can be a complex process involving both solidification dynamics and concentration phase separation. In many situations long-lived "eutectic" structures form, i.e., morphologies containing alternating rods or lamella of the two crystal phases. The fine scale of these morphologies (typically much smaller than a micron) gives them useful mechanical properties, and makes them quite valuable in casting and welding processes. To understand the complex morphologies that occur in eutectic solidification, it is imperative to understand the nonequilibrium dynamics used in their creation. In a previous paper [1] a phase field model was used to describe the dynamics of the solidification of an undercooled liquid at the eutectic concentration under isothermal conditions. This work led to the prediction of an interesting crossover scaling behavior, from diffusion-limited growth to lamellar growth to phase segregation, and is consistent with preliminary experiments on the crystallization of amorphous Fe-B compounds.

The purpose of this paper is examine the more general situation of nonisothermal crystallization. For simplicity an isolated sample with material properties and conditions that lead to a uniform, but time dependent temperature field, will be considered. These conditions should be satisfied for the crystallization of glassy or amorphous metals, most of which contain a eutectic point. Examples include $\mathrm{Fe}-\mathrm{B}, \mathrm{Cu}-\mathrm{Zr}$, and $\mathrm{Mg}-\mathrm{Zn}$ systems. In these metallic materials the thermal diffusivities should be large enough to relax "instantaneously" any spatial variations in temperature field created by latent heat production at the amorphous-crystal fronts. This work will focus on the crystallization of such amorphous materials. In Sec. II a quantitative criteria for the applicability of this uniform temperature approximation will be presented and discussed for the Fe-B system.

It is well known that the production of latent heat during solidification or crystallization can lead to interesting dynamical behavior. A classic example is the destabilization of a liquid-solid $(L-S)$ front (i.e., the Mullins-Sekerka $[2,3]$ instability), that can lead to dendritic structures. In explosive crystallization $[4,5]$ the production of latent heat can lead to oscillations in the amorphous-crystal $(A-X)$ front velocity, and in principle to a period doubling sequence to chaos. In both examples the interesting behavior is specifically related to spatial gradients in the temperature field (or more precisely the diffusion of the latent heat generated at the $A-X$ or $L-S$ interfaces). In eutectic crystallization, the lamella spacing is related to the $A-X$ velocity, which is in turn related to the undercooling (or "overheating"). Thus, even though the temperature field in this work will be assumed to be uniform in space, its time dependence will lead to interesting complications. At a minimum these nonisothermal conditions will destroy the lamellar growth regime (in which crystal droplets grow in size by increasing the length and number, but not width, of lamella) seen in the isothermal case.

To understand the extremely complex process of nonisothermal homogeneous eutectic nucleation of glassy metals, two simplified scenarios will first be considered. First, the change in lamellar thickness with time will be isolated by considering the invasion of a one dimensional lamellar front into a supercooled amorphous matrix in the absence of thermal fluctuations. This analysis simplifies the dynamics by excluding the competition and coalescence of neighboring fronts that is common in nucleation. Second, the process of nonisothermal crystallization via homogeneous nucleation will be considered in the absence of a eutectic reaction. In this manner, the dynamics of nonisothermal crystallization can be decoupled from the eutectic reactions and used as a reference point.

The outline of this paper is as follows. In Sec. II a general dynamical model of nonisothermal eutectic crystallization will be presented. A limit will be derived for which this general model reduces to one in which the temperature field is spatially uniform. This limit will provide a criteria for the applicability of this model in terms of nucleation rates, ther- 
mal diffusivities, and growth velocities. Section III will focus on simple nonisothermal nucleation in the absence of a eutectic reaction. Expressions will be derived for the time dependence of the crystal volume fraction for the two cases considered in Sec. IV on eutectic crystallization, the first being a one dimensional front and the second homogeneous nucleation. Finally in Sec. V section some brief conclusions will be given.

\section{DYNAMICAL MODEL}

In order to describe a eutectic structure, at least two fields must be introduced, one $(\psi)$ to distinguish between the amorphous and crystal phases and another $\left(c \equiv C-C_{E}\right.$, i.e., concentration deviation from the eutectic concentration) to distinguish between the two crystal phases. In principle the order parameter describing the two crystal phases should not be a simple scalar quantity; however, as was shown in a previous paper [1], the model presented is consistent with the standard sharp interface models used by many others [7-10]. and recovers many well known results for directional eutectic growth. The model can be represented by two dynamical equations

$$
\partial c / \partial t=\vec{\nabla} \cdot M_{c} \vec{\nabla} \delta \mathcal{F}\{c, \psi\} / \delta c+\eta_{c}
$$

and

$$
\partial \psi / \partial t=-M_{\psi} \delta \mathcal{F}\{c, \psi\} / \delta \psi+\eta_{\psi},
$$

where $\mathcal{F}$ is the free energy functional, and can be approximated by

$$
\mathcal{F}\{c, \psi\}=\int d \vec{r}\left(f(c, \psi)+\frac{\kappa_{\psi}}{2}|\vec{\nabla} \psi|^{2}+\frac{\kappa_{c}}{2}|\vec{\nabla} c|^{2}\right) .
$$

The bulk free energy $(f)$ can be written $f(c, \psi)=-r \psi^{2} / 2+u \psi^{4} / 4+\left(\alpha \Delta T-\beta c^{2}\right) \psi+b c^{4} / 4$, where $\Delta T=\left(T-T_{m}\right) / T_{E}, T_{m}$ being the melting temperature at $\beta=0$, and $T$ the temperature. All the parameters that enter $\mathcal{F}$ will be considered to be independent of temperature. In this formulation no distinction is made between an amorphous solid and a frozen liquid. The temperature dependence of $M_{c}$ and $M_{\psi}$ will be incorporated at a later stage. The phase diagram for Eq. (3) is shown in Fig. 1.

For nonisothermal conditions an equation of motion for the temperature field must be specified. The dynamics of the temperature field are in general controlled by diffusion, Newtonian cooling, and latent heat production. Thus the equation for the dimensionless undercooling $(\Delta T)$ is

$$
\frac{\partial \Delta T}{\partial t}=\mu\left(\Delta T_{0}-\Delta T\right)+D \nabla^{2} \Delta T+\frac{\mathcal{L}}{2} \frac{\partial \bar{\psi}}{\partial t},
$$

where $\mu$ describes the coupling to a heat bath at an undercooling $\Delta T_{0}, D$ is the thermal diffusivity, $\mathcal{L}=H /\left(c_{p} T_{E}\right)$, $H$ is the enthalpy of fusion, and $c_{p}$ is the specific heat at constant pressure and $\bar{\psi}=\psi /|\psi|$. For this work an isolated solid is considered (i.e., $\mu=0$ ).

If the latent heat liberated during the crystallization (i.e., as $\psi$ evolves in time) can be 'instantaneously' distributed throughout the sample, $\Delta T$ is constant in space and equal to

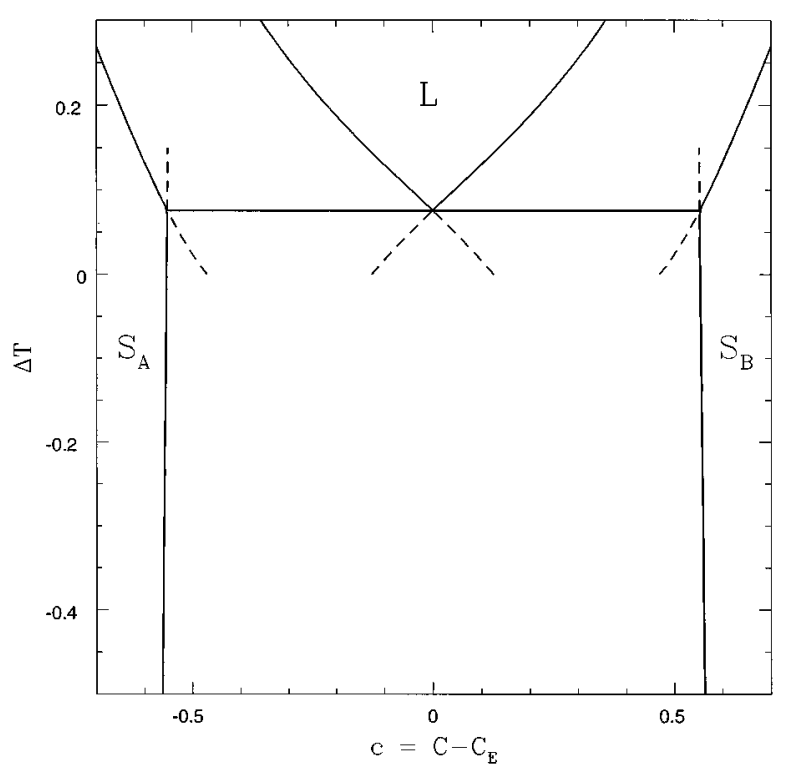

FIG. 1. Phase diagram obtained by minimization of the eutectic free energy presented in the text. The regime enclosing the letter $L$ corresponds to a liquid phase. The regimes enclosing the letters $S_{A}$ and $S_{B}$ correspond to solid phases of different concentrations. The other enclosed regions correspond to the coexistence of phases.

$$
\Delta T(t)=\Delta T(0)+\mathcal{L} \mathcal{V}(t)
$$

where $\mathcal{V}$ is the crystal volume fraction. To estimate the validity of this approximation it is useful to consider a single droplet with an infinitely thin interface growing at constant velocity $(v)$, i.e.,

$$
\partial \Delta T / \partial t=D \nabla^{2} \Delta T+\mathcal{L} v \delta\left(r-\left[v t+R_{0}\right]\right),
$$

where $R_{o}$ is the initial droplet size and the temperature is uniform at $t=0$. For the uniform temperature approximation to be valid, the temperature must relax before the interface has moved a significant distance, i.e., of the order of a correlation length. Thus it is required that the temperature relaxes for times $t \approx \zeta_{\psi} / v$. Thus it is appropriate to consider the sharp interface limit $v t \approx \zeta_{\psi} \ll R_{0}$. In this limit the solution to Eq. (6) in Fourier space is

$$
\Delta \hat{T}(\vec{q}, t) \approx e^{-D q^{2} t} \Delta \hat{T}(\vec{q}, 0)+\mathcal{L}\left(\frac{v R_{o}^{d-1}}{L^{d}}\right) \Omega_{d}\left(\frac{e^{i \gamma}-e^{-D q^{2} t}}{i \gamma+D q^{2}}\right)
$$

where $\quad \gamma=v q \cos (\theta), \quad \Omega_{1}=1, \quad \Omega_{2}=\int_{0}^{2 \pi} d \theta, \quad$ and $\Omega_{3}=\int_{0}^{\pi} d \theta \int_{-\pi}^{\pi} d \phi \sin (\theta)$. The parameter $L$ is the 'longest' length scale in the problem. From a practical point of view $L$ can be interpreted as several times the average distance between nucleation sites, or in the final crystallized sample several times the average crystallite size. For $q=0$ the solution is $\hat{T}(0, t)=\hat{T}(0,0)+\mathcal{L} \mathcal{V}$, which is consistent with Eq. (5). For $q \neq 0$, the first term on the right-hand side of Eq. (7) is negligible if $D q^{2} t \gg 1$. A very conservative estimate of this condition can be obtained by setting $q=2 \pi / L$, i.e., 


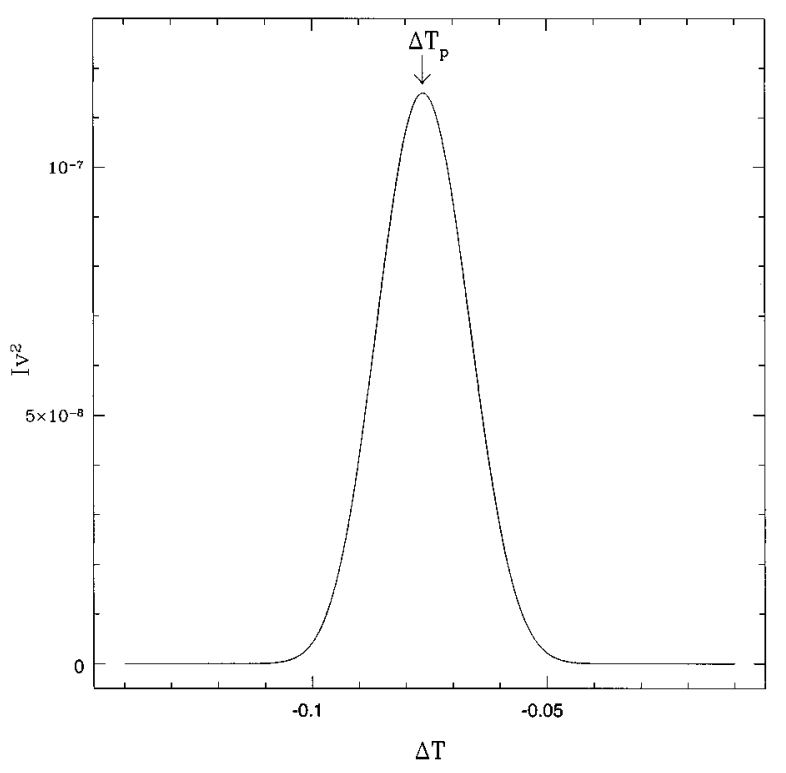

FIG. 2. Crystalization rate dependence on temperature in two dimensions.

$$
(2 \pi)^{2} D /(L v) \gg L / \zeta_{\psi}
$$

For the second term to be negligible, it must also be small compared to 1 (in the dimensionless units used here), which implies $(2 \pi)^{2} D /(L v) \gg\left(R_{c} / L\right)^{d-1} \mathcal{L}$. Since $R_{c} \gg \zeta_{\psi}$, and $\mathcal{L}$ is of order 1, this condition is satisfied if Eq. (8) is satisfied.

As an example, consider an $\mathrm{Fe}-\mathrm{B}$ system. At low temperatures (i.e., $500 \mathrm{~K}<T<800 \mathrm{~K}$ ) the droplet velocity is of the order $10^{-7}<v<10^{-2} \mu \mathrm{m} / \mathrm{s}$ [6], and the thermal diffusivity is of the order $D \approx 10^{8} \mu \mathrm{m}^{2} / \mathrm{s}$. Assuming the correlation length is a few lattice spaces (i.e, $\zeta \approx 10^{-3} \mu \mathrm{m}$ ) Eq. (8) reduces to $L \ll 10^{4} \rightarrow 10^{6} \mu \mathrm{m}$. For experiments in the temperature range stated above, this limit should be satisfied.

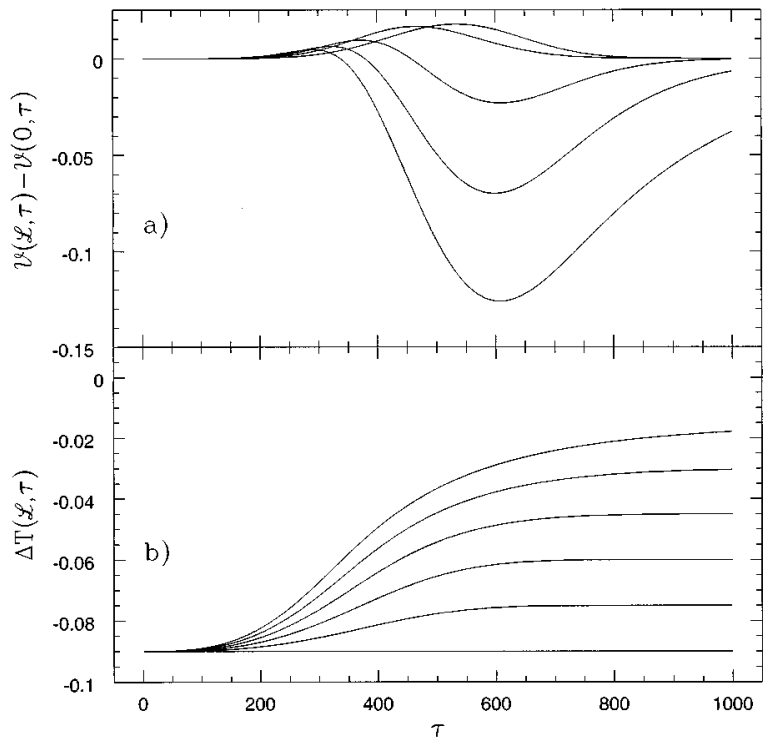

FIG. 3. Dynamics of volume fraction and undercooling. In (a) the lines from top to bottom at $\tau=600$ correspond to $\mathcal{L}=0.015$, $0.03,0.045,0.06$, and 0.075 . In (b) the lines from bottom to top correspond to $\mathcal{L}=0.015,0.03,0.045,0.06$, and 0.075 .
For conditions under which these assumptions are valid, $\Delta T$ rises uniformly, and Eqs. (1) and (2) can be simplified since $M_{c}(T)$ and $M_{\psi}(T)$ are now independent of position. It is convenient to eliminate $M_{\psi}(T)$ by rescaling time by the following variable transformation $\tau=\int_{0}^{t} d t^{\prime} M_{\psi}\left(T\left(t^{\prime}\right)\right) r$. It is also useful to transform to the following dimensionless variables $\phi=\psi / \psi_{0}, h=c / c_{0}, \vec{x}=\vec{r} / \zeta_{\psi}$, and $T^{\prime}=T / T_{0}$, where $\psi_{0}=(r / u)^{1 / 2}, \quad c_{0}=\left(2 \beta \psi_{0} / b\right)^{1 / 2}, \quad \zeta_{\psi}=\left(\kappa_{\psi} / r\right)^{1 / 2}, \quad$ and $T_{0}=\psi_{0} r / \alpha \approx 4 T_{E}$. These substitutions lead to

$$
\begin{gathered}
\partial h / \partial \tau=R_{t} / R_{L}^{2} \nabla^{2}\left(h^{2}-\phi-R_{L}^{-2} \nabla^{2}\right) h+\nu_{h}, \\
\partial \phi / \partial \tau=\left(1-\phi^{2}+\nabla^{2}\right) \phi+\beta^{\prime} h^{2}-\Delta T^{\prime}+\nu_{\phi}, \\
\Delta T^{\prime}(\tau)=\Delta T_{o}^{\prime}+\mathcal{L}^{\prime} \mathcal{V}, \\
\left\langle\nu_{h}(\vec{x}, \tau) \nu_{h}\left(\vec{x}^{\prime}, \tau^{\prime}\right)\right\rangle=2 \gamma \epsilon_{\phi} \nabla^{2} \delta\left(\tau-\tau^{\prime}\right) \delta\left(\vec{x}-\vec{x}^{\prime}\right),
\end{gathered}
$$

and

$$
\left\langle\nu_{\phi}(\vec{x}, \tau) \nu_{\phi}\left(\vec{x}^{\prime}, \tau^{\prime}\right)\right\rangle=2 \epsilon_{\phi} \delta\left(\tau-\tau^{\prime}\right) \delta\left(\vec{x}-\vec{x}^{\prime}\right) .
$$
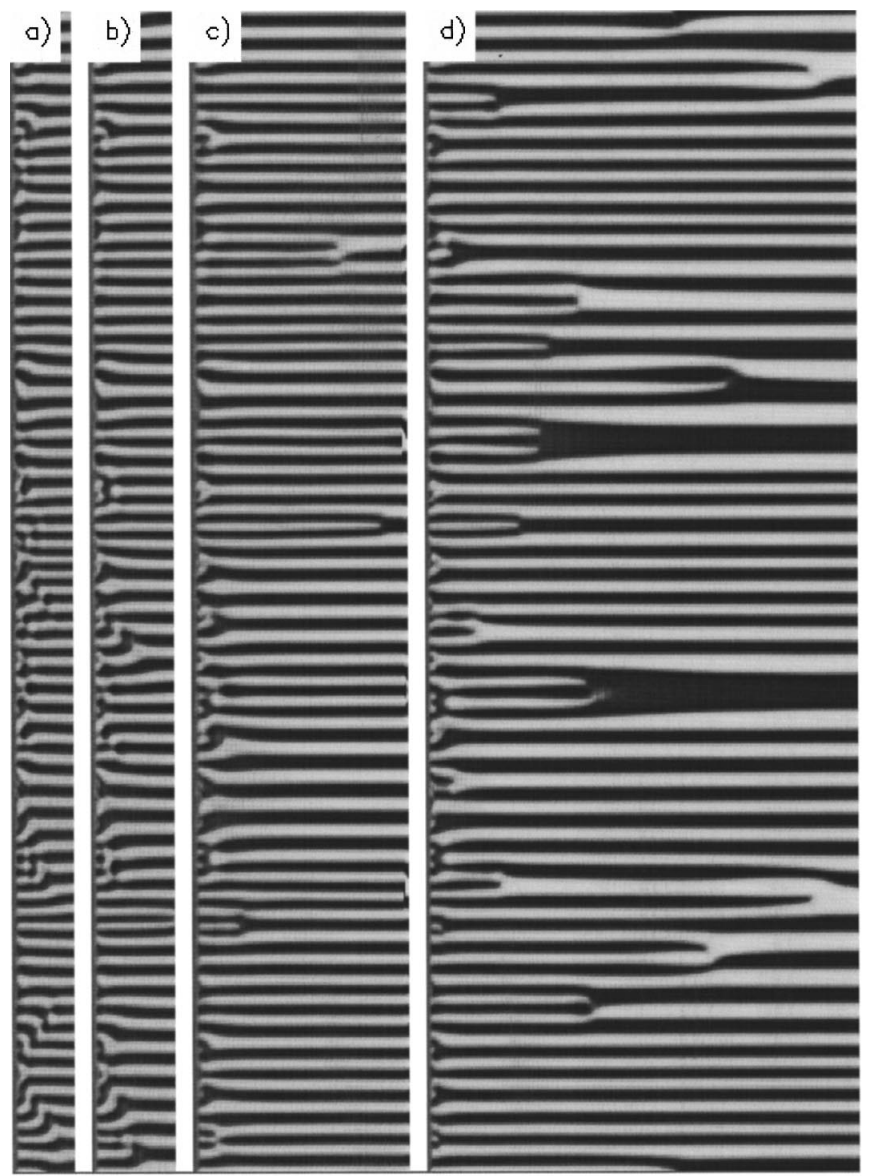

FIG. 4. Time dependence of the $A-X$ front. In this figure the concentration field $(h)$ is shown at a distance of three lattice sites behind the $A-X$ front as a function of time. In (a), (b), (c), and (d), the front is shown for $\mathcal{L}=0.0,0.0075,0.015$, and 0.0225 , respectively. The total time evolved for each of these simulations was $3600,4800,12500$, and 25000 for $\mathcal{L}=0.0,0.0075,0.015$, and 0.0225 , respectively. 


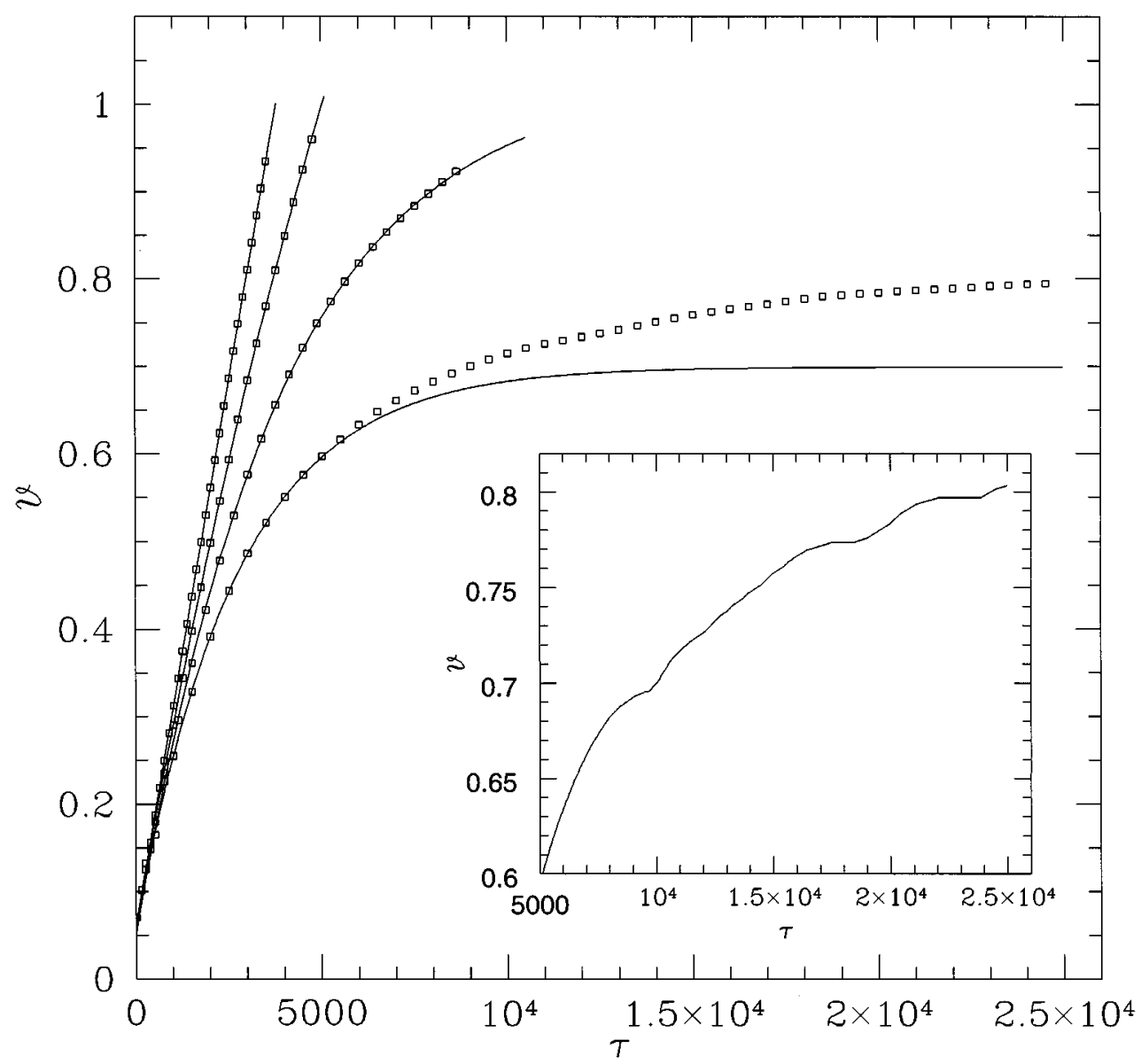

FIG. 5. Dynamics of the volume fraction. The points correspond to the numerical results and the lines to fits to Eq. (16). From left to right the lines correspond to $\mathcal{L}=0.0,0.0075,0.015$, and 0.0225 . In the inset an individual run is shown for $\mathcal{L}=0.0225$.

The various dimensionless parameters in these equations are $\epsilon_{\phi}=u k_{B} \psi_{0} \zeta_{\psi}^{d} / r, \gamma=\left[b r /(2 \beta)^{2}\right]\left[R_{t} / R_{L}^{2}\right], R_{t}=\Gamma_{\psi} / \Gamma_{c}$, $R_{L}=\zeta_{\psi} / \zeta_{c}, \quad \zeta_{c}=\left(\kappa_{c} / 2 \beta \psi_{0}\right)^{1 / 2}, \quad \beta^{\prime}=2 \beta^{2} / b r, \quad \mathcal{L}^{\prime}=\mathcal{L} / T_{0}$, $\Gamma_{\psi}=1 / M_{\psi} r$, and $\Gamma_{c}=\zeta_{c}^{2} / 2 M_{c} \beta \psi_{0}$. For simplicity in the following pages the prime superscripts will be dropped, and $R_{T}$ will be taken to be independent of temperature.

The model is now complete, and can be used to describe nonisothermal eutectic nucleation. Before considering these complex dynamics the influence of nonisothermal conditions will be considered in the absence of the eutectic interaction.

\section{NONISOTHERMAL CRYSTALLIZATION}

When an amorphous material is heated above the glass transition, small crystallites nucleate in and grow into the amorphous background. As latent heat is generated both the nucleation rate $(I)$ and the droplet velocity $(v)$ are altered. For the dimensionless model presented here [i.e., where $M_{\psi}(\Delta T)$ has been scaled into $\left.\tau\right]$ the increase in temperature will always decrease $v$, but may increase or decrease $I$, since $I$ goes to zero at $T=0$ and at $T=T_{m}$ (or $T=T_{E}$ for a eutectic), and has a peak somewhere in between. It should be noted that when the velocity is transformed into physical units [by the relationships given above Eq. (9)] it will also go to zero at zero temperature. The basic functional dependences of $I$ and $v$ on $T$ are captured by most theories, al- though an accurate calculation of $I$ is a formidable task. For the purposes of this paper the temperature dependence of $I$ will be simply taken from the approximate formula of Langer and Turski [12].

To begin these calculations consider Eq. (10) in the absence of concentration, i.e.,

$$
\partial \phi / \partial \tau=\left(1-\phi^{2}+\nabla^{2}\right) \phi-\Delta T+\nu_{\phi}
$$

and Eq. (11). For a single spherical droplet it is quite simple to calculate $\mathcal{V}(\tau)$. The rate of growth of this droplet can be calculated by expanding around a planar solution, i.e., $\phi(\vec{r}, t) \approx \phi^{1 D}[r-R(\tau)]$, where $\phi^{1 d}$ satisfies $\left(\partial^{2} / \partial x^{2}+1-\phi^{2}\right) \phi=0$, and is given by $\phi^{1 d}=\tanh (x / \sqrt{2})$. This gives the following equation for the radius $[R(\tau)]$ of the droplet:

$$
\partial R / \partial \tau=v-(d-1) / R
$$

where $v=\Delta T \Delta \phi / \sigma, \sigma=\int d x\left(\partial \phi_{o} / \partial x\right)^{2}$ is the surface tension, and $\Delta \phi$ is the miscibility gap. The critical droplet size for this model is defined by $\partial R(\tau) / \partial \tau=0$ and is $R_{c}=(d-1) \sigma / \Delta T \Delta \phi$. The undercooling can be introduced through Eq. (11), and the volume fraction is related to the droplet radius by the relationship $\mathcal{V}(\tau)=\Omega_{d} R^{d} / d$. In one dimension the solution is 


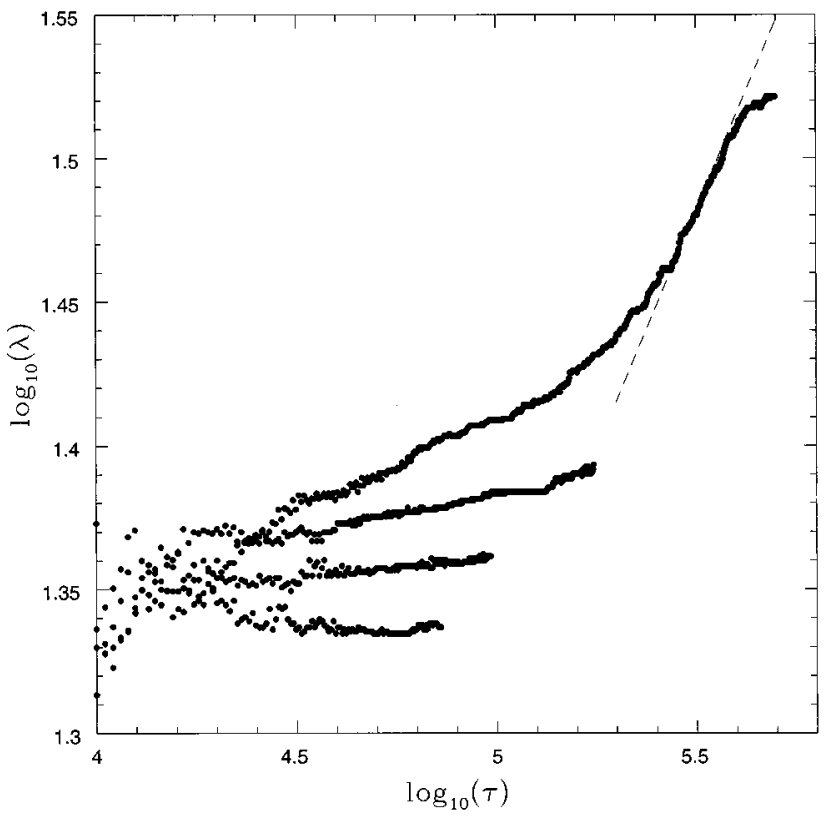

FIG. 6. Dynamics of the lamella wavelength. The points from bottom to top correspond to $\mathcal{L}=0.0075,0.015$, and 0.0225 . The dashed line has a slope of $\frac{1}{3}$.

$$
\mathcal{V}_{1 D}(\tau)=\frac{|\Delta T(0)|}{\mathcal{L}}+\left(\mathcal{V}_{1 \mathrm{D}}(0)-\frac{|\Delta T(0)|}{\mathcal{L}}\right) e^{(-\mathcal{L}|\Delta \phi| \tau / L \sigma)}
$$

This solution can be compared with the growth of a onedimensional eutectic front presented in Sec. IV.

For nucleation phenomena the standard form for the volume fraction [13] is

$$
\mathcal{V}(\tau)=1-\exp \left(-\frac{\Omega_{d}}{d} \int_{0}^{\tau} d \tau^{\prime} I\left(\tau^{\prime}\right) R^{d}\left(\tau, \tau^{\prime}\right)\right)
$$

where $I(\tau)$ is the nucleation rate, and $R\left(\tau, \tau^{\prime}\right)$ is the radius of a droplet at time $\tau$ that was nucleated at time $\tau^{\prime}$. In order to evaluate $R\left(\tau, \tau^{\prime}\right)$ it is standard to take the limit $R \gg R_{c}$ such that $R\left(\tau, \tau^{\prime}\right)=\int_{\tau^{\prime}}^{\tau} d \tau^{\prime \prime} v\left(\tau^{\prime \prime}\right)$. In all subsequent calculations this limit will be assumed. Once a form for $I(\tau)$ is specified, Eq. (17) combined with Eqs. (15) and (11) form a closed set of equations. For example, for heterogeneous nucleation, where $I(\tau)=I_{0} \delta(\tau)$, the set of equations is simply $\mathcal{V}(\tau)=1-\exp \left[-\left[\Omega_{d} / d\right] I_{0} R(\tau, 0)^{d}\right]$ and $\partial R / \partial \tau=\Delta \phi\left[\Delta T_{0}+\mathcal{L} \mathcal{V}\right] / \sigma$.

For homogeneous nucleation the situation is more complicated, since the nucleation rate is a strong function of temperature. To understand these dynamics the nucleation rate will be taken to be [12] $I=K \Omega_{0} \exp \left(-\Delta F / K_{b} T\right)$, where $K$ is the dynamic prefactor, $\Omega_{0}$ is the statistical prefactor, and $\Delta F$ is the free energy difference between the metastable state and the saddle point configuration (i.e., a critical droplet). In what follows, it will be assumed that the nucleation rate depends on time only through the temperature field. It should be noted that even under isothermal conditions, there is a period of time needed for the nucleation rate to reach its asymptotic value [14]. This effect is sometimes taken into account by introducing an offset time.
Relying on the work of Langer and Turski [12] it is straightforward to determine that $K=(d-1) / R_{c}^{2}, \Omega_{0}$ $=L^{d}\left[2 R_{c}^{2} /(d-1)\right]^{1 / 2}\left[S_{c}^{d} \sigma\right]^{d / 2}$, and $\Delta F=\Omega_{d} R_{c}^{d-1}\left(2 R_{c} \Delta T /\right.$ $d+\sigma)$, where $S_{c}^{d}$ is the surface "area" of the $d$ dimensional critical droplet, and $L$ is the system size. For isothermal conditions $\mathcal{V}(t)=1-\exp \left[-\left[\Omega_{d} / d(d+1)\right] I v^{d} t^{d+1}\right]$. The important factor determining the dynamics of the $\mathcal{V}$ is the product $I v^{d}$. This quantity has a peak somewhere between $T=0$ and $T=T_{m}$, which will be denoted $\Delta T_{p}$. A plot of this quantity is shown in Fig. 2 for a two-dimensional system. Using these approximations $\mathcal{V}(t)$ can determined as a function of $\mathcal{L}$ by solving Eqs. (17), (15), and (11) selfconsistently in the limit $R \gg R_{c}$.

To illustrate the influence of $\mathcal{L}$ on the crystallization dynamics, $\mathcal{V}(\mathcal{L}, t)$ was determined for a number of values of $\mathcal{L}$, starting at $\Delta T$ slightly lower than $\Delta T_{p}$ [i.e., $\Delta T(0)$ $=-0.09]$. In Fig. 3 the difference, $\Delta \mathcal{V}(\mathcal{L}, t)=\mathcal{V}(\mathcal{L}, t)$ $-\mathcal{V}(0, t)$, is shown as a function of time and $\mathcal{L}$. The basic shape of $\Delta \mathcal{V}(\mathcal{L}, t)$ can be easily inferred from the temperature dependence of $I v^{2}$ as shown in Fig. 2. For $\mathcal{L}=0.015$, the final undercooling is -0.075 [i.e., $\Delta T(\infty)=\Delta T(0)+\mathcal{L}$ ] which is just slightly above $\Delta T_{p}$. Thus, for this $\mathcal{L}$, the quantity $I v^{2}$ is always increasing and consequently $\Delta \mathcal{V}(0.015, t)>0$. For larger values of $\mathcal{L}$, the undercooling becomes sufficiently larger that $\Delta \mathcal{V}(\mathcal{L}, t)$ becomes negative at later times. These analytic calculations will be compared with the numerical simulations of eutectic nucleation and growth. Although the process of eutectic nucleation is more complicated, the simple nonisothermal effects included in this section must be present. Eutectic nucleation is complicated by the fact that the "temperature field" that enters Eq. (14) is concentration dependent [see Eq. (10)].

\section{NONISOTHERMAL LAMELLAR GROWTH}

When an amorphous metal is heated above the glass transition and near the eutectic concentration, small crystallites emerge and concentration segregation occurs. In concert with these dynamics heat is generated and the nucleation and crystallite growth rates change. These changes significantly alter the subsequent crystallization (as was seen in Sec. III) and phase segregation processes. To observe these effects, the dynamical model described by Eqs. (9)-(13) was numerically simulated on a two-dimensional discrete square lattice. Details of the numerical procedures were given in a previous paper [1].

For simplicity, the influence of a changing growth rate will be studied in the absence of nucleation by considering a one-dimensional crystal front invading an amorphous background in the absence of thermal fluctuations (i.e, $\epsilon_{\phi}=0$ ). For these simulations the parameter set was $\left(R_{L}, R_{t}, \beta, \Delta T(0), \epsilon_{\phi}\right)=(1,1,0.015,-0.03,0)$, and the mesh size and time step were $\Delta x=1.3$ and $\Delta \tau=0.05$ respectively. These simulations were initialized such that for $x<10 \Delta x$ $(x>10 \Delta x) \quad \phi=1 \quad(\phi=-1) \quad$ in a system of size $\left(L_{x}, L_{y}\right)=(128 \Delta x, 1024 \Delta x)$. The propagation of this front was then examined for different values of $\mathcal{L}$. In these and all subsequent simulations the temperature is evolved according to Eq. (11), where the volume fraction is numerically evaluated at each time step. 

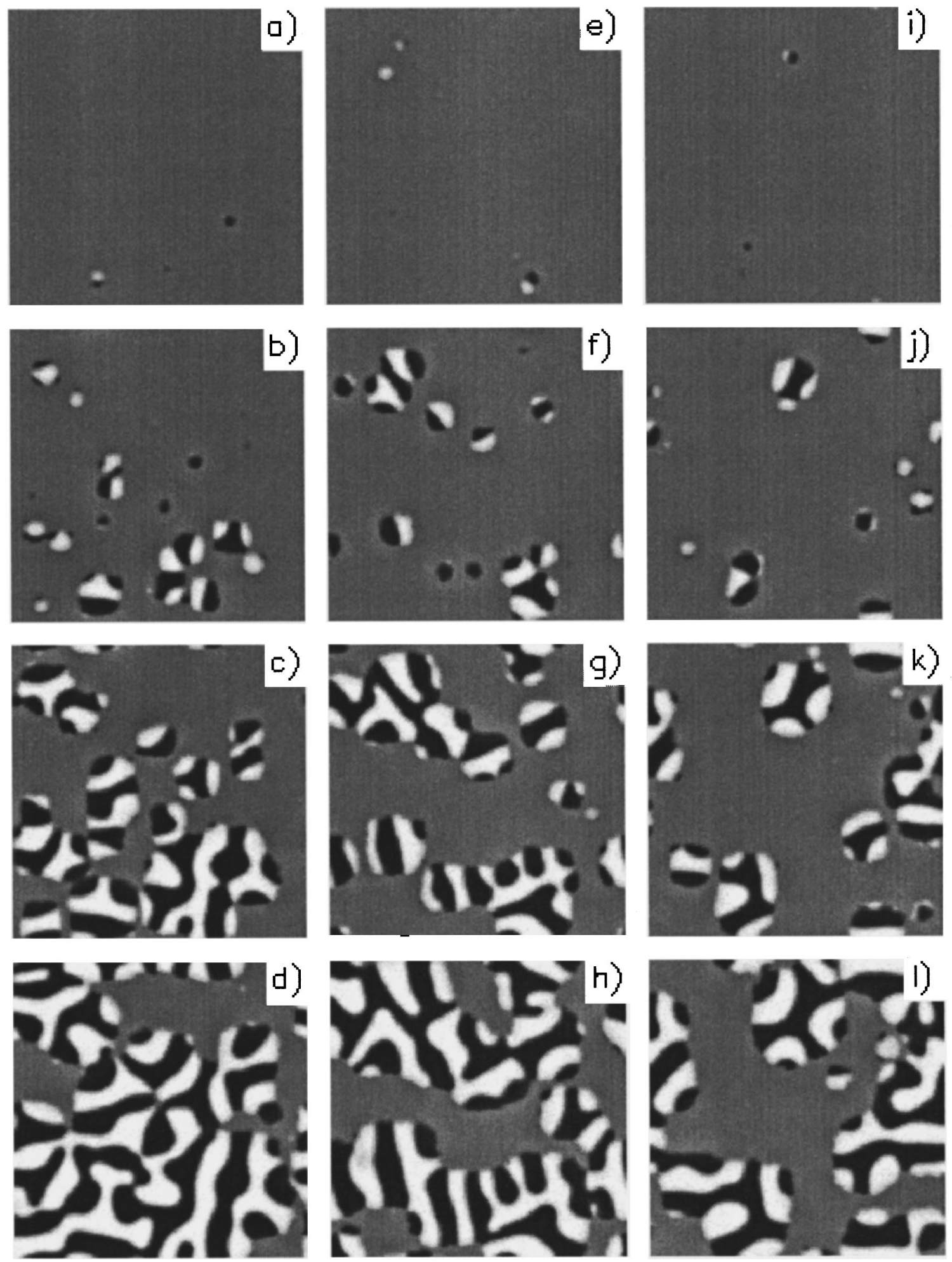

FIG. 7. Time and spatial dependence of the concentration field. (a) $-(\mathrm{d})$ correspond to $\mathcal{L}=0.015$ for times $\tau=125,250,375$, and 500. (e) and (f) and (i) - (l) correspond to $\mathcal{L}=0.045$ and 0.075 , respectively, for the same times in (a) $-(\mathrm{d})$.

For $\mathcal{L}=0$, the $A-X$ interface moves at a constant velocity and the lamella wavelength is quickly selected. For $\mathcal{L}>0$ the velocity of the interface slows down and gradually the wavelength increases as smaller lamella are eliminated. These dynamics are shown in Fig. 4 for $\mathcal{L}=0.0,0.0075,0.015$, and 0.0225 . In this figure the dark and light stripes correspond to the two different solid phases, and the gray region to the amorphous solid. The elimination of the lamella can be easily seen for the largest value of $\mathcal{L}$. Various statistics were averaged over at least ten independent runs, and are shown in Figs. 5 and 6. The dynamics of $\mathcal{V}$ (see Fig. 5) are consistent with the form given in Eq. (16) (i.e., $A-B e^{-C t}$ ), but the specific values of $A, B$, and $C$ are not consistent with the parameters of the simulations. In essence the value of $\mathcal{L}$ 


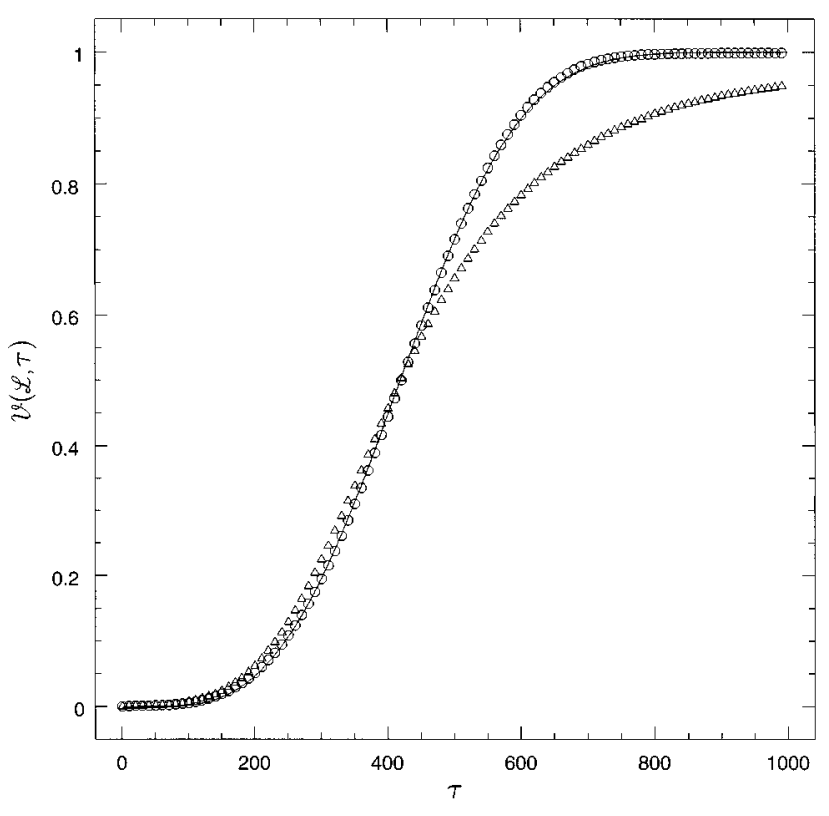

FIG. 8. Time dependence of the volume fraction. The open circles correspond to $\mathcal{L}=0.0$ and the open triangles to $\mathcal{L}=0.3$. The solid line is a fit to the form $\mathcal{V}=1-\exp \left[-\pi I v^{2}\left(t-t_{0}\right)^{3}\right]$, where $I v^{2}=4.34 \times 10^{-9}$ and $t_{0}=48.74$.

seems to be much too large in the fits, implying that the fronts will die out before the sample temperature reaches $T_{E}$. This is most apparent at the largest value of $\mathcal{L}$, in which the form breaks down near $\tau=500$. The averaged quantities shown is this figure tend to obscure an interesting feature. At $\mathcal{L}=0.00225$, for $\tau>500$, the interface seems to move in a "jerky" motion. An example of this motion is shown for an individual run in the inset of Fig. 5. This motion is due to the elimination of lamella, which occur at discrete intervals.

In Fig. 6 the time dependence of the average lamella wavelength $(\lambda)$ is displayed on a logarithmic scale. This figure clearly shows that $\lambda$ increases with time, implying that $\lambda$ increases with decreasing velocity (similar to directional eutectic growth $[1,7,11])$. The growth of $\lambda$ must be consistent with known growth laws for conserved fields (since the concentration is a conserved field). For conserved fields, typical length scales grow at a rate of $t^{1 / 3}$; thus $\lambda$ should grow at this rate in the asymptotic limit. While this limit was not examined in this paper, the results are consistent with a $t^{1 / 3}$ growth at the latest times observed.

\section{NONISOTHERMAL EUTECTIC NUCLEATION}

The basic result of these calculations is that, as the front heats up, the interface slows down and the lamella spacing increases. This should also play an important role in the homogeneous nucleation of the amorphous metal. For these simulations the system was initialized in an amorphous state with random fluctuations in $\psi$ with the parameter set $\left(R_{L}, R_{t}, \beta, \Delta T(0), \gamma, \epsilon_{\phi} \hat{T}\right)=(1.2,1,0.0055,-0.06,0.01$, $2 / 3)$, where $\hat{T}=(1+\Delta T / 0.15)$ (so that "zero" temperature is at $\Delta T=-0.15$ ). For $\mathcal{L}=0.0,0.015,0.03,0.045,0.06$, and 0.075 , a system of size $256 \times 256$ mesh points was simulated, and statistics were averaged over 15 runs.

A qualitative assessment of the influence of $\mathcal{L}$ can be

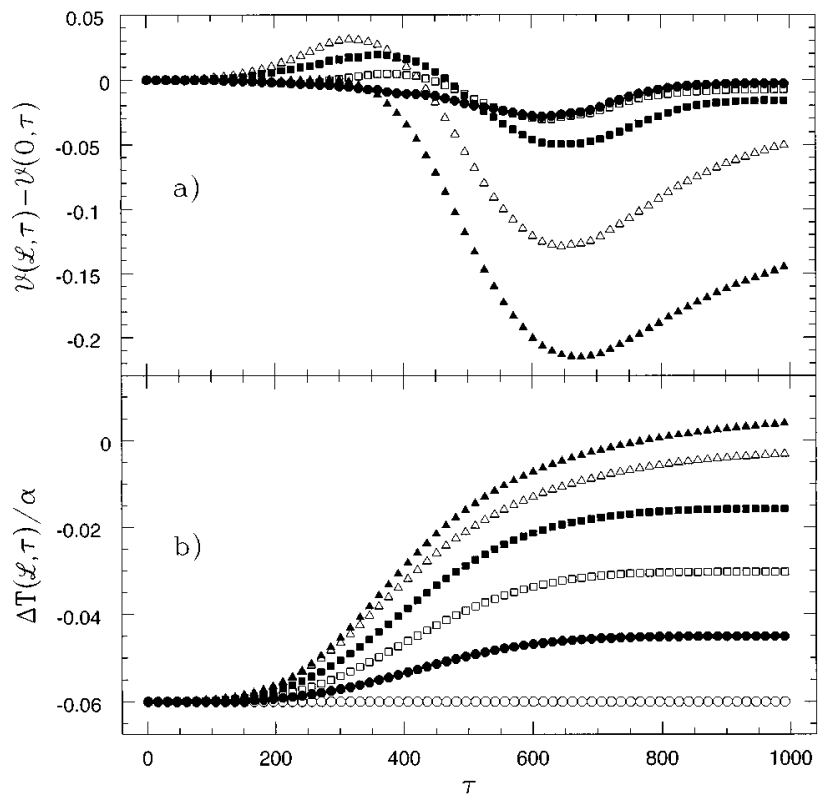

FIG. 9. Dynamics of the volume fraction and undercooling. In (a) the dynamics of $\mathcal{V}(\mathcal{L}, t)-\mathcal{V}(0, t)$ is shown while in (b) the dynamics of the undercooling is displayed. In both figures the open circles, and solid circles, open squares, solid squares, open triangles, and solid triangles correspond to $\mathcal{L}=0.0,0.015,0.03,0.045$, 0.06 , and 0.075 , respectively.

obtained by a visual examination of the time dependence of the concentration field. In Fig. 7 the concentration field is displayed at several different times and values of $\mathcal{L}$. In this figure the gray matrix corresponds to the metastable amorphous phase and the drops contain the two solid phases. For a more quantitative picture of the crystallization process, the volume fraction is shown in Fig. 8 for $\mathcal{L}=0.0$ and 0.045 . The isothermal case can be fit to the form $\mathcal{V}=1-\exp \left\{-[\pi / 3] I v^{2}\left(\tau-\tau_{0}\right)^{3}\right\}, \quad$ where $I v^{2} \approx 1.3 \times 10^{-8}$ and $\tau_{0} \approx 49$. The fact that an offset time is needed to fit the data implies that the thermal fluctuations did not immediately relax to their equilibrium value.

For a closer examination of $\mathcal{V}$ the deviations from the isothermal limit are shown in Fig. 9(a). In Fig. 9(b) the undercooling is shown as a function of time for all values of $\mathcal{L}$. These figures can be compared with the calculations for noneutectic crystallization shown in Fig. 3. Clearly the general trends for eutectic crystallization are quite similar to noneutectic crystallization. Nevertheless differences between the two cases exist. For example at $\mathcal{L}=0.015, \Delta V$ decreases, which contradicts the small $\mathcal{L}$ behavior seen in Fig. 3. This effect can probably be attributed to the time needed for the fluctuations to relax as the temperature rises. If $T$ is fixed and $\Delta T$ is increasing, the quantity $I v^{2}$ always decreases. Thus if the "temperature" that enters the Boltzmann factor in $I$ (i.e., $e^{-\Delta F / k_{B} T}$ ) lags the "true" temperature, the volume fraction will slow down in comparison to the isothermal case.

In Fig. 10 the dynamics of the average concentration spacing $[R(\mathcal{L}, \tau)]$ is shown, where $R$ is defined to be the first zero of the pair correlation function $[G(r, \tau)$ $\left.\equiv \int d \theta\left\langle h\left(\vec{r}^{\prime}, \tau\right) h\left(\vec{r}+\vec{r}^{\prime}, \tau\right)\right\rangle\right]$. For clarity each curve has been offset, except in the inset, where a direct comparison be- 


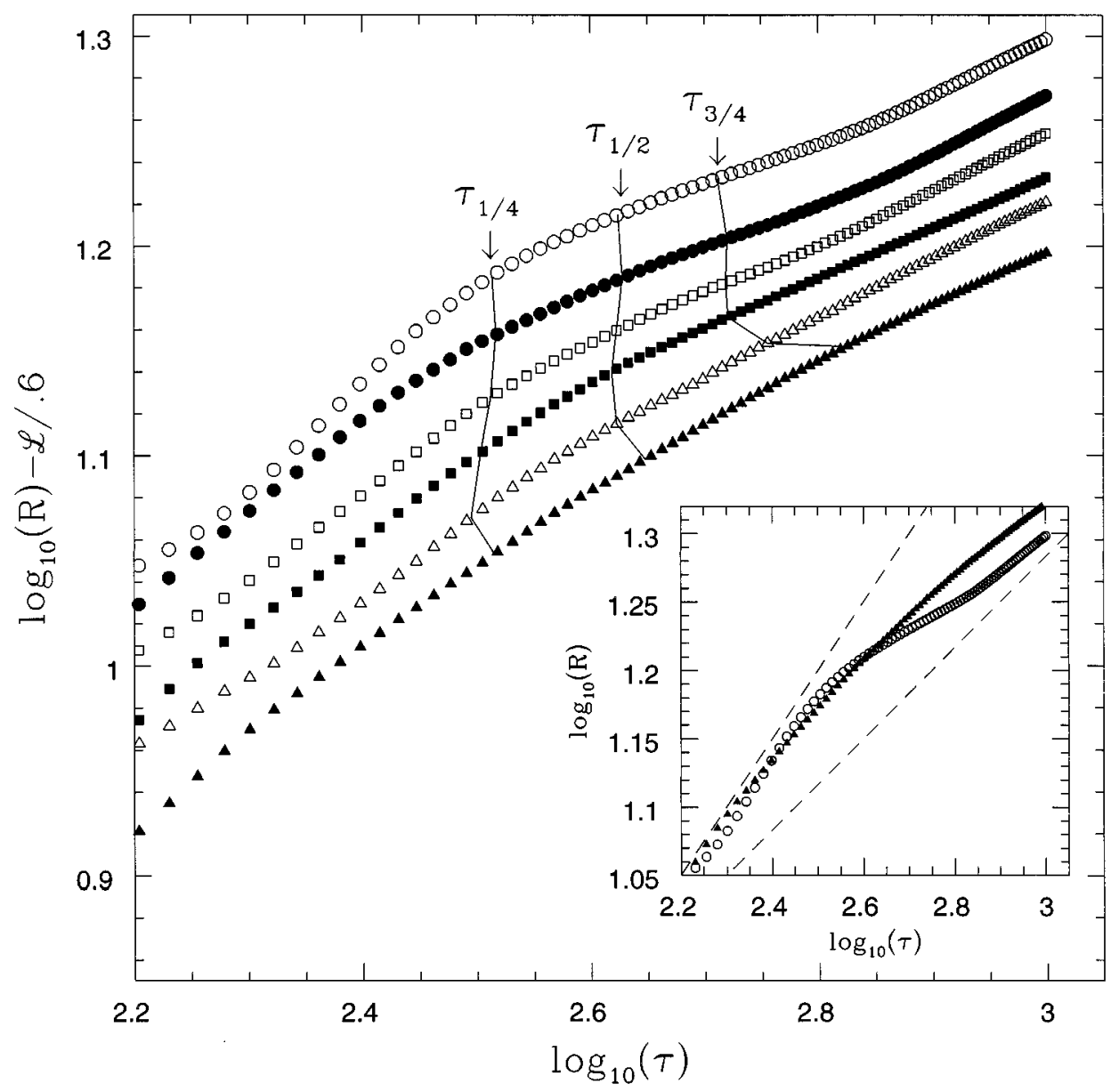

FIG. 10. Time dependence of the average domain size. For convenience the values of $\ln (R)$ have been separated by 0.025 for each value of $\mathcal{L}$. The symbols are the same as in Fig. 8. The lines denoted $\tau_{1 / 4}, \tau_{1 / 2}$, and $\tau_{3 / 4}$ correspond to the times needed to crystallize $25 \%$, $50 \%$, and $75 \%$ of the sample, respectively. In the inset a direct comparison between $\mathcal{L}=0.0$ and $=0.075$ is shown. The dashed lines correspond to slopes of $\frac{1}{2}$ (upper line) and $\frac{1}{3}$ (lower line).

tween $R(0.0, \tau)$ and $R(0.075, \tau)$ is shown. To interpret these results it is instructive to recapitulate the results discussed in [1] for $\mathcal{L}=0$. For this case there is an initial period of diffusive growth as the small nucleated drops attempt to grow to the lamella wavelength by absorbing atoms from the surrounding amorphous background. This regime leads to $R$ $\propto t^{1 / 2}$. When $R$ reaches the lamella wavelength (dictated by the $A-X$ velocity), $R$ should become fixed in time (i.e., the "lamellar' growth regime), except for small corrections due to additional droplets nucleating and growing. This regime is difficult to distinguish in the present simulations, since the droplets begin to coalesce shortly after the diffusive regime. There is, however, a small regime (between $25 \%$ and $50 \%$ crystallization) in which $R$ slows considerably. Finally once the system is crystallized, spinodal decomposition occurs for which $R \propto t^{1 / 3}$.

For $\mathcal{L}=0.03$, this pattern is roughly repeated, but as $\mathcal{L}$ approaches 0.075 there is no evidence of diffusive or lamellar growth regimes. Since the lamella wavelength increases as the temperature increases, there can be no regime in which $R$ is constant. This is especially apparent for $\mathcal{L}=0.075$, which shows that as $R(0, t)$ begins to slow down $R(0.075, t)$ continues increasing, so that when the spinodal decomposition regime is entered the value of $R(0.075, t)$ is larger than $R(0, t)$.

In general there are many factors influencing the dynamics of $R$. In particular as the undercooling goes to zero both the critical droplet size and lamellar wavelength diverge. In addition, the nucleation rate changes drastically.
Thus it is extremely difficult, and probably unrealistic, to identify different growth regimes, the exception being the final regime in which spinodal decomposition dominates. Asymptotically, it should be expected that $R \propto t^{1 / 3}$ for all $\mathcal{L}<\Delta T_{E}-\Delta T(0)$.

\section{CONCLUSION}

The dynamics of nonisothermal homogeneous eutectic nucleation is a complex process involving many different growth processes. It seems unlikely that generic scaling arguments, used in many domain growth phenomena [15], will be applicable to this phenomena. Nevertheless many of the observations described in this work should be experimentally accessible in the crystallization of metallic glasses.

\section{ACKNOWLEDGEMENTS}

K.R.E. would like to thank Nigel Goldenfeld for many useful discussions. This work was supported by Grant No. NSF-DMR-9596202. We would also like to acknowledge support of the Pittsburgh Supercomputing Center and the Lehigh University Computing Center. K.R.E. acknowledges the support of Grant No. NSF-DMR-89-20538, administered through the University of Illinois Materials Research Laboratory. 
[1] K. R. Elder, F. Drolet, J. M. Kosterlitz, and M. Grant, Phys. Rev. Lett. 72, 677 (1994).

[2] W. W. Mullins and R. F. Sekerka, J. Appl. Phys. 35, 444 (1964).

[3] J. S. Langer, Rev. Mod. Phys. 52, 1 (1980).

[4] W. van Saarloos and J. D. Weeks, Physica 12D, 279 (1984).

[5] D. A. Kurtze, W. van Saarloos, and J. D. Weeks, Phys. Rev. B 30, 1398 (1984).

[6] U. Köster and U. Herold, in Glassy Metals I, edited by H.-J. Guntherodt and H. Beck (Springer-Verlag, New York, 1981).

[7] K. A. Jackson and J. D. Hunt, Trans. Metall. Soc AIME 236, 843 (1966); 236, 1129 (1966).

[8] J. S. Langer, Phys. Rev. Lett 44, 1023 (1980); V. Datye and J. S. Langer, Phys. Rev. B 24, 4155 (1981).

[9] K. Kassner and C. Misbah, Phys. Rev. Lett. 65, 1458 (1990); 66, 445 (1991); Phys. Rev. A 44, 6513 (1991); 44, 6533 (1991).

[10] A. Karma, Phys. Rev. Lett. 59, 71 (1987); Phys. Rev. E 49, 2245 (1994).
[11] This conclusion cannot be directly inferred from Jackson and Hunt's [7] work, since a "minimum undercooling assumption" is made. For a uniform temperature field no such minimization can be made. Nevertheless, if these arguments are extended to minimize the distance between the $A-X$ front and the concentration front, the same velocity-wavelength relationship is obtained.

[12] J. S. Langer and L. A. Turski, Phys. Rev. A 8, 3230 (1983).

[13] A. N. Kolmogorov, Bull. Acad. Sci. USSR, Phys. Ser. 3, 335 (1938).

[14] K. F. Kelton, A. L. Greer, and C. V. Thompson, J. Chem. Phys. 79, 6261 (1983).

[15] J. D. Gunton, M. San Miguel, and P. Sahni, in Phase Transitions and Critical Phenomena, edited by C. Domb and J. L. Lebowitz (Academic, London, 1983), Vol. 8, p. 267;

J. D. Gunton and M. Droz, Introduction to the Theory of Metastable and Unstable States (Springer-Verlag, Berlin, 1983). 